\title{
Comparison of a Metronome-guided Prehospital Medication Infusion Technique with Manual Calculation: A Simulated Randomized, Controlled, Cross-over Study
}

\section{Samuel Galvagno ( $\sim$ sgalvagno@som.umaryland.edu )}

University of Maryland, Baltimore

James Cloepin

Anne Arundel Fire Department

Jeannie Hannas

Anne Arundel Fire Department

Kurt Rubach

University of Maryland Baltimore Washington Medical Center

Andrew Naumann

Maryland Institute of Emergency Medical Services Systems

Jonathan Wendell

University of Maryland Baltimore Washington Medical Center

\section{Research Article}

Keywords: prehospital, emergency medical services, medication infusion, medical calculation, medication dosing, resuscitation

Posted Date: April 28th, 2021

DOl: https://doi.org/10.21203/rs.3.rs-380767/v1

License: (c) (1) This work is licensed under a Creative Commons Attribution 4.0 International License. Read Full License 


\title{
Comparison of a metronome-guided prehospital medication infusion technique with manual calculation: a simulated randomized, controlled, cross-over study
}

\author{
Samuel M Galvagno, DO, PhD, FCCM \\ (CORRESPONDING AUTHOR) \\ Professor \\ Department of Anesthesiology \\ Program in Trauma \\ University of Maryland School of Medicine \\ 22 S Greene Street, T5S18 \\ Baltimore, MD 21201 \\ sgalvagno@som.umaryland.edu \\ 410-328-0256 \\ Assistant Medical Director \\ Anne Arundel County Fire Department \\ ORCID ID: 0000-0001-5563-4092
}

\section{James Cloepin, NRP}

Anne Arundel County Fire Department

Millersville, MD 21108

fd000993@aacountry.org

\section{Jeannie Hannas, NRP}

Anne Arundel County Fire Department

Millersville, MD 21108

fd001383@aacountry.org

\section{Kurt S. Rubach, RN, NRP}

Nurse Manager

Baltimore-Washington Medical Center

Glen Burnie, MD 21061

kurt.s.rubach@gmail.com

Andrew Naumann, NRP, EMT-T

Maryland Institute for Emergency Medical Services Systems (MIEMSS)

653 West Pratt Street

Baltimore, MD 21201

anaumann@miemss.org

Jonathan Wendell, MD, FACEP

Clinical Assistant Professor

University of Maryland Baltimore Washington Medical Center

Department of Emergency Medicine

Glen Burnie, MD 21061

Medical Director

Anne Arundel County Fire Department

Fd009824@aacountry.org 


\section{ABSTRACT}

\section{Background}

Limited research regarding administration of timed medication infusions in the prehospital environment has identified wide variability with accuracy, timing, and overall feasibility. This study was a pragmatic, randomized, controlled, crossover study comparing two different techniques for medication infusion administration. We hypothesized that the use of a metronome-based technique would decrease medication dosage errors and reduce time to administration for intravenous medication infusions.

\section{Methods}

Forty-two nationally registered paramedics were randomized to either a metronome-based technique versus a standard stopwatch-based technique. Each subject served as a control. Subjects were asked to establish an infusion of amiodarone at a dose of $150 \mathrm{mg}$ administered over 10 minutes, simulating treatment of a hemodynamically stable patient with sustained monomorphic ventricular tachycardia. Descriptive statistics and multiple linear regression were used for data analysis.

\section{Results}

When compared to a standard stopwatch-based technique, a metronome-based technique was associated with faster time to goal, fewer mid-infusion adjustments, and greater ease of use.

\section{Conclusions}

Use of a metronome technique for establishing medication infusion rates may help prehospital clinicians provide safer and more precise medication delivery.

KEYWORDS: prehospital, emergency medical services, medication infusion, medical calculation, medication dosing, resuscitation 


\section{DECLARATIONS}

\section{Ethics Approval and Consent to Participate}

All methods were carried out in accordance with relevant guidelines from the University of Maryland School of Medicine (HP-00096185). All experimental protocols were approved by the University of Maryland School of Medicine. This study was deemed exempt (the need for approval was waived) by the University of Maryland School of Medicine Institutional Review Board. Institutional review board oversight was not required under $45 C F R 46.104$ as the aim of this research was to the effectiveness of an instructional technique for calculating medication infusion rates in a prehospital environment. Informed consent was not required for this study since participants were volunteers and no identifiable information was collected.

\section{Consent for Publication}

Not applicable.

\section{Availability of Data and Materials}

The datasets generated and/or analysed during the current study are located in the METRONOMEEMS repository persistent web link to datasets: https://www.dropbox.com/s/3uz6rh4xz70p56z/metronome data.dta?dl=0.

\section{Competing Interests}

Dr. Galvagno reports personal fees from Northwest Anesthesia Seminars, grants from the Department of Defense, and is an employee of the United States Air Force Reserve. However, nothing in this work is related to this employment. The views expressed are those of the author and do not reflect the views of the US Air Force, the Department of Defense, or US Government.

The remaining authors have no competing interests to disclose.

\section{Funding}


No funding was required for this study. Materials used were donated by the Anne Arundel County Fire Department (expired materials). This work was not funded by an external agency.

\section{Authors' Contributions}

SMG conceived the study design, performed data collection, completed all data analysis and interpretation, and drafted the article.

JW conceived the study design, performed data collection, and critically revised the article.

$\mathrm{JC}, \mathrm{JH}, \mathrm{AN}$ and KSR conceived the study design, performed data collection, and critically revised the article.

\section{Acknowledgements}

The authors thank Fire Chief Trisha L. Wolford, Battalion Chief Robert Vaccaro, and Deputy Chief Timothy Mikules for supporting this study. 


\section{Introduction}

Delivery of intravenous (IV) infusions of fluid and medications is a challenging but foundational skill for prehospital clinicians. ${ }^{1}$ An infusion pump is preferred to ensure appropriate dosing, but such devices are expensive, require extensive training, and are not available in many EMS systems. Even when using intravenous infusion flow regulators, deviations from expected infusion volumes are common and potentially harmful. ${ }^{2,3}$ Conventional calculation of prehospital IV infusion rates requires a mathematical computation based on the medication dose, delivery interval, and number of drops in the IV administration set per volume. Thus, a method to mitigate the difficulty and potential for error when administering prehospital IV infusions could assist EMS clinicians by facilitating accurate infusion control.

As the scope of prehospital medicine continues to evolve, the ability to quickly and safely administer IV medication infusions will remain an essential skill to enhance patient outcomes. For example, in patients with refractory ventricular fibrillation, early administration of amiodarone has been associated with improved neurological outcomes for some out-of-hospital cardiac arrest (OHCA) patients. ${ }^{5,6}$

The objective of this study was to assess the effectiveness, accuracy, and ease of use of a metronome-guided IV medication infusion technique. We hypothesized that when compared to conventional prehospital IV medication infusion techniques, the metronome technique would be associated with fewer errors and decreased time to target infusion rate. 


\section{Methods}

Nationally registered paramedics (NRP) from Anne Arundel County Fire Department, Maryland, USA, served as subjects during this study. This study was deemed exempt from institutional review board oversight (HP-00096185) at the University of Maryland School of Medicine since the aim of this research was to test the simulated effectiveness of an instructional technique for calculating medication infusion rates in a prehospital environment. The study was conducted in a stationary ambulance, where participants were asked to correctly establish an intravenous infusion rate using one of two techniques. Using a random number generator, subjects were initially randomized to either a "standard" group using micro drippers and a calculation or a "metronome" group using an audible cadence to establish the correct medication infusion rate. Each subject served as her or his own control; the order of medication infusion rate performance was reversed after the initial assigned technique was complete. Primary outcomes of interest included time to target infusion rate (from start to finish), time to goal medication infusion rate, and total elapsed time. Ease of use was evaluated with a 5-point Likert scale with a value of " 1 " indicating most difficult and "5" indicating easiest.

Subjects were asked to establish an infusion of amiodarone at a dose of $150 \mathrm{mg}$ administered over 10 minutes, simulating treatment of a hemodynamically stable patient with sustained monomorphic ventricular tachycardia. Multiple IV administration sets (15-, 20-, and 60 drips/mL) were used to simulate restocking from different receiving hospitals. The amiodarone vial $(3 \mathrm{~mL})$ was injected into a $100 \mathrm{~mL}$ IV bag of $5 \%$ dextrose in water. Subjects were required to use the following formula to establish the correct infusion rate (drops): 


\section{$=$ Flow Rate (drops/min)}

\section{Formula 1. Intravenous infusion set calculation.}

For example, using a volume of $103 \mathrm{~mL}$ of amiodarone, at 20 drips $/ \mathrm{mL}$, the flow rate for a 10-minute infusion was $206 \mathrm{gtts} / \mathrm{min}$. Once the flow rate was calculated, subjects were instructed to establish an infusion of amiodarone using either the standard or metronome technique according to the randomization scheme. For the standard technique, a watch was used to synchronize the drops/min. In the metronome group, a Quik Time ${ }^{\circledR}$ metronome (John Hornby Skews \& Co., Leeds, UK) was used. Depending on the drips/min per the infusion set used, the metronome cadence was adjusted to match the desired flow rate.

Descriptive statistics and multiple linear regression (with appropriate regression diagnostics) were used for data analysis. Using a one-way ANOVA to detect a statistically significant difference of 2 minutes between techniques, at an alpha of 5\% and with $80 \%$ power, a total of 12 participants were required in each group. All tests were two-tailed and a $P$ value of $<0.005$ was considered statistically significant. ${ }^{7}$ Analyses were performed in Stata version 15 (Stata Corp, College Station, TX) and GraphPad Prism 7.0d (GraphPad Software, La Jolla, CA). 


\section{Results}

Forty-two NRPs were enrolled (Figure 1).

\section{|CONSORT Diagram}

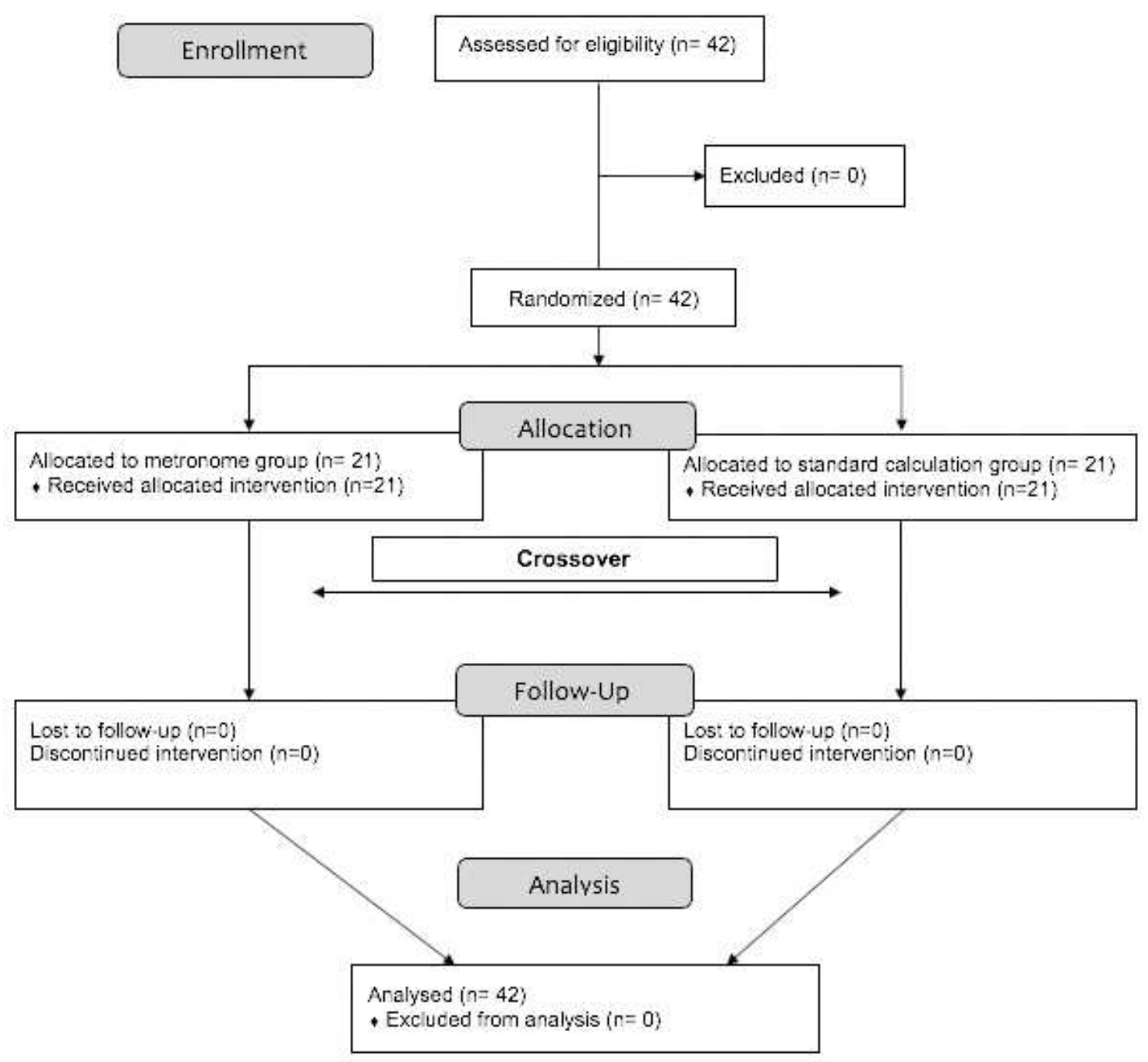

Figure 1. CONSORT study flow diagram.

The mean number of years each subject had worked as an NRP was 10.4 (6.7). Twelve (14.6\%) NRPs had prior experience working for critical care transport organizations, none were registered nurses, and two (2.4\%) were physician's assistants.

In the standard group, adjustments to the drip rate were required $63 \%$ of the time; in the metronome groups, adjustments were required in $34.4 \%(P=0.14)$. Infusions were 
finished prematurely $9.8 \%$ of the time in the standard group vs. $4.9 \%$ of the time in the metronome group $(P=0.29)$. No adjustments were required in $56 \%$ of the standard group and in $75.6 \%$ of the metronome group. $(P=0.06)$.

Time to infusion completion was not significantly different between the groups (standard group, mean time $460.9 \mathrm{~s}$ [118.7 s] vs. metronome group, mean time $510.8 \mathrm{~s}$ $[116 \mathrm{~s}] ; P=0.19)$. Median time to goal was significantly faster in the metronome group (median $34 \mathrm{~s}$; IQR, 22-54 s) compared to the standard group (median $50 \mathrm{~s}$; IQR 38-61 s) $(P=0.006)$ (Figure 2).

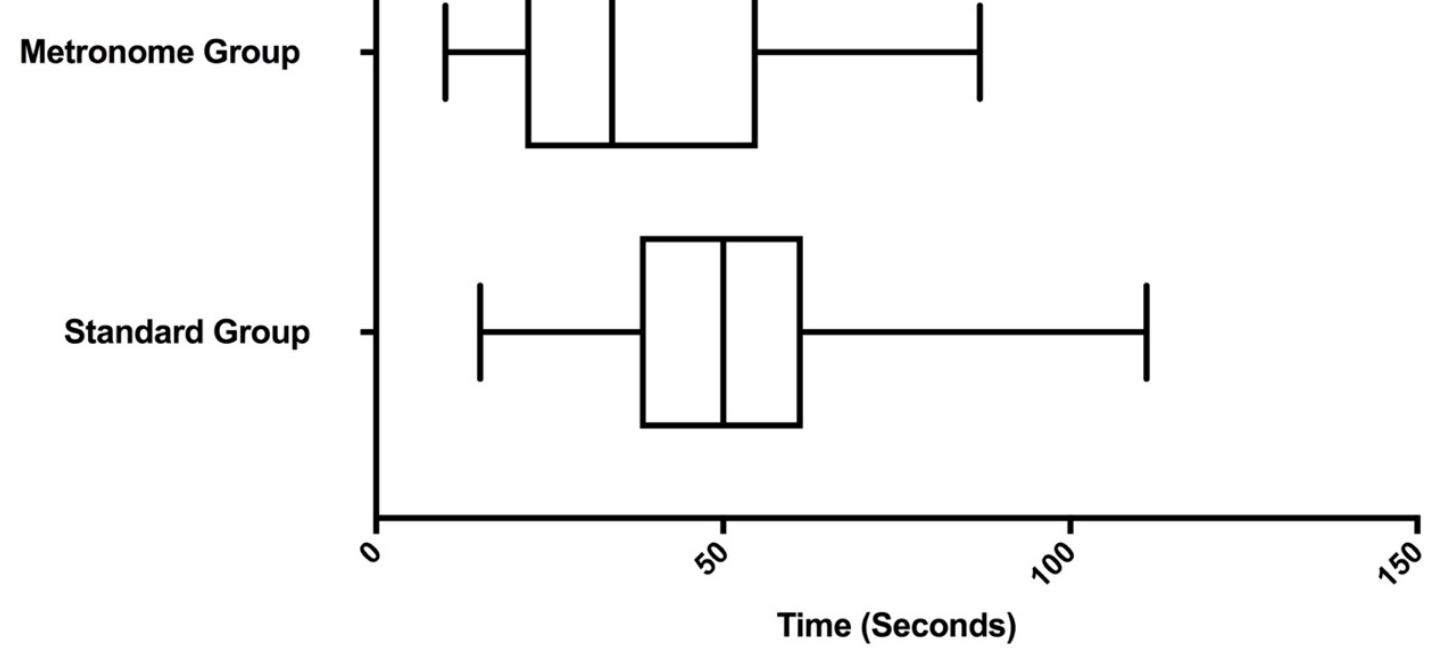

Figure 2. Box and whisker plots depicting median time to infusion rate goal. $P=0.006$.

When controlling for total years of experience and critical care transport experience with multiple linear regression, time to goal infusion rate was significantly faster in the metronome group (mean of 13 second faster; 95\% confidence interval, 22-7 seconds; $P$ $<0.001)$. Ease of use was reported to be significantly higher for the metronome group (median ranking 5, IQR 4-5) compared to the standard group (median ranking 2, IQR 23; $P<0.001$ ) (Figure 3). 


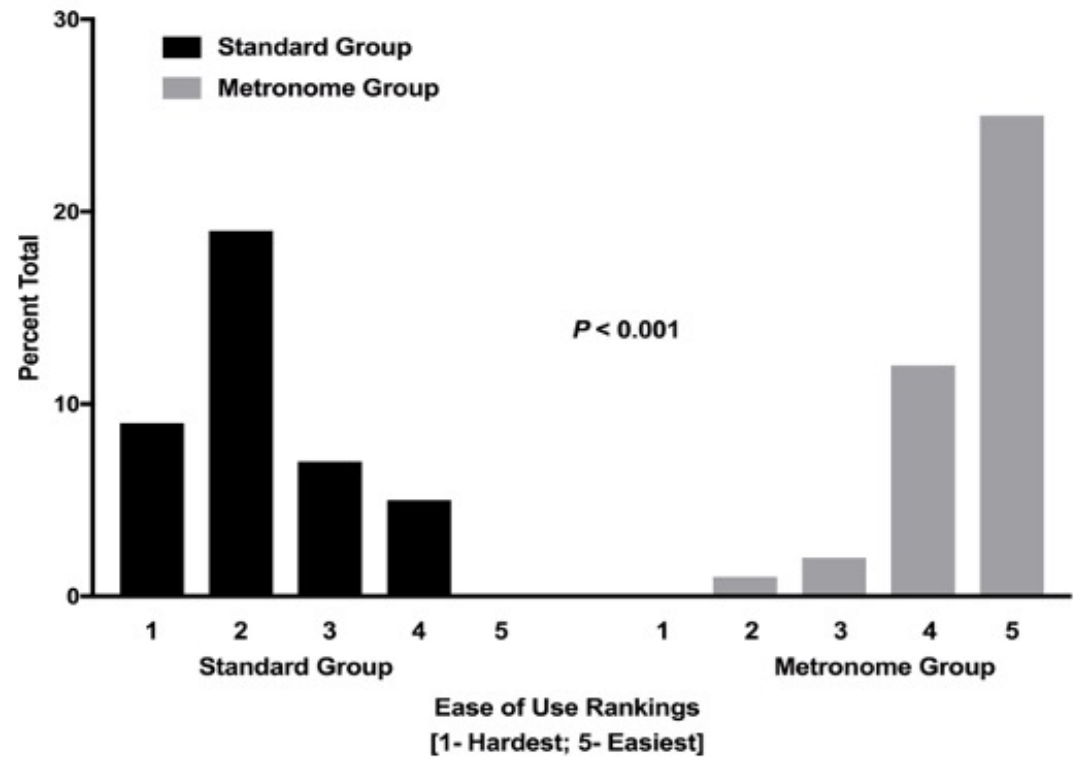

Figure 3. Self-reported ease of use. 


\section{Discussion}

Administration of intravenous medication infusions in the prehospital arena is an arduous task. Even when using intravenous infusion flow regulators, significant deviations from volume and flow rates are possible, leading to potential medical delivery errors. ${ }^{2}$ When delivering fluid volumes alone, accuracy may be highly variable. ${ }^{4}$ In this randomized, cross over study, use of a metronome was associated with greater ease of use and shorter time to goal infusion rate.

The results of this study have significant implications for prehospital clinicians. Infusions of lidocaine and amiodarone may be associated with clinically relevant outcomes, and proper dosing is essential. In one large randomized double-blind study, ${ }^{8}$ both of these agents have been associated with improved survival to hospital admission, ${ }^{6}$ and amiodarone has been shown to be moderately effective for the treatment of sustained ventricular tachycardia..$^{5}$ Amiodarone is also associated with improved neurological outcome at hospital discharge for out-of-hospital cardiac arrest in patients who had ventricular fibrillation as the presenting dysrhythmia. ${ }^{9}$

The prehospital literature is largely devoid of data describing safe and efficient practices for medication infusion delivery. Park et al. compared four methods of infusion control in a cohort of emergency medicine nurses, highlighting the inaccuracy of dial flow controllers and supporting the use of an "intravenous infusion therapy helper" based on a metronome and drop counter. ${ }^{10}$ A phone application with a metronome-like flow rate control function was used control the volume of a fluid infusion. Compared to a stopwatch technique, the volume of fluid was more precise with the metronome-based technique, although an additional control was added using a control function within the application to add additional precision. The outcome of interest in this study was fluid volume, not medication dosing. In our study, use of an off-the-shelf metronome 
demonstrated superior effectiveness and ease of use. Metronome applications for smartphones are prevalent, free, and can be used in the field to adjust medication flow rates. Such techniques could prove useful for EMS jurisdictions administering infusions that require timing, such as amiodarone, lidocaine, magnesium, epinephrine, and others.

There are a number of limitations to our work. The crossover nature of this study may have introduced confounding due to carryover between technique assignments since the metronome technique might have helped the participants remember a correct drip cadence. Although the metronome group had a lower overall number of premature infusion completions, compared to the standard technique, this difference was not statistically significantly different, likely due to the sample size. Although randomized and administered in a simulated prehospital setting, this study did not involve actual patients and only one medication infusion was assessed. It is possible that other medication infusions may be subject to a greater or lesser degree of error.

\section{Conclusion}

Precise control of prehospital medication infusions is required to prevent harm and maximize therapeutic effectiveness. As many EMS jurisdictions across the world do not have access to electronic infusion pumps, novel techniques are required for training and clinical practice. In this study, the use of a metronome technique was associated with faster time to goal infusion rate and greater ease of use compared to standard techniques. 


\section{References}

1. National Registry of Emergency Medical Technicians. Paramedic Candidate Handbook. Columbus, OH: National Registry of Emergency Medical Technicians; 2019.

2. Loner C, Acquisto NM, Lenhardt H, et al. Accuracy of Intravenous Infusion Flow Regulators in the Prehospital Environment. Prehosp Emerg Care 2018;22:645-9.

3. Coppler PJ, Padmanabhan R, Martin-Gill C, Callaway CW, Yealy DM, Seymour CW. Accuracy of Prehospital Intravenous Fluid Volume Measurement by Emergency Medical Services. Prehosp Emerg Care 2016;20:125-31.

4. Asaithambi G, Chaudhry SA, Hassan AE, Rodriguez GJ, Suri MF, Qureshi AI. Adherence to guidelines by emergency medical services during transport of stroke patients receiving intravenous thrombolytic infusion. J Stroke Cerebrovasc Dis 2013;22:e42-5.

5. Foerster CR, Andrew E, Smith K, Bernard S. Amiodarone for sustained stable ventricular tachycardia in the prehospital setting. Emerg Med Australas 2018;30:694-8.

6. McLeod SL, Brignardello-Petersen R, Worster A. Comparative effectiveness of antiarrhythmics for out-of-hospital cardiac arrest: a systematic review and network meta-analysis. Resuscitation 2017;121:90-7.

7. Wasserstein RLM, Lazar NA. The ASA's statement on P-values: context, process, and purpose. Am Stat 2016;70:129-33.

8. Kudenchuk PJ, Brown SP, Daya M, et al. Amiodarone, lidocaine, or placebo for out-of-hospital cardiac arrest. N Engl J Med 2016;374:1711-22.

9. Lee DK, Kim YJ, Kim G, et al. Correction to: Impact of early intravenous amiodarone administration on neurological outcome in refractory ventricular fibrillation: retrospective analysis of prospectively collected prehospital data. Scand J Trauma Resusc Emerg Med 2020;28:23.

10. Park K, Lee J, Kim S, et al. Infusion volume control and calculation using metronome and drop counter based intravenous infusion therapy helper. Int J Nurs Pract 2013;19:257-64. 
Figures

\section{|CONSORT Diagram}

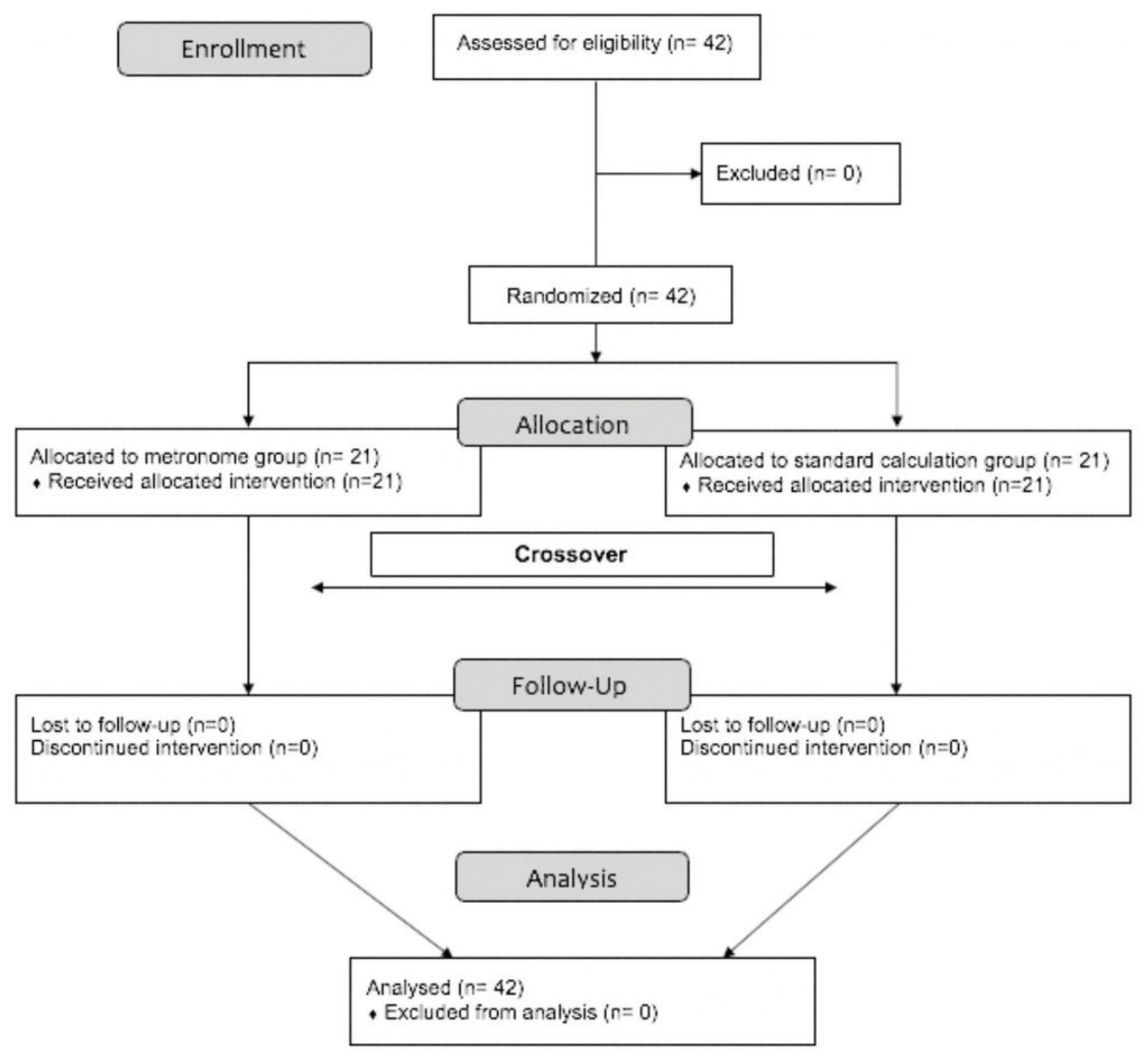

Figure 1

CONSORT study flow diagram. 


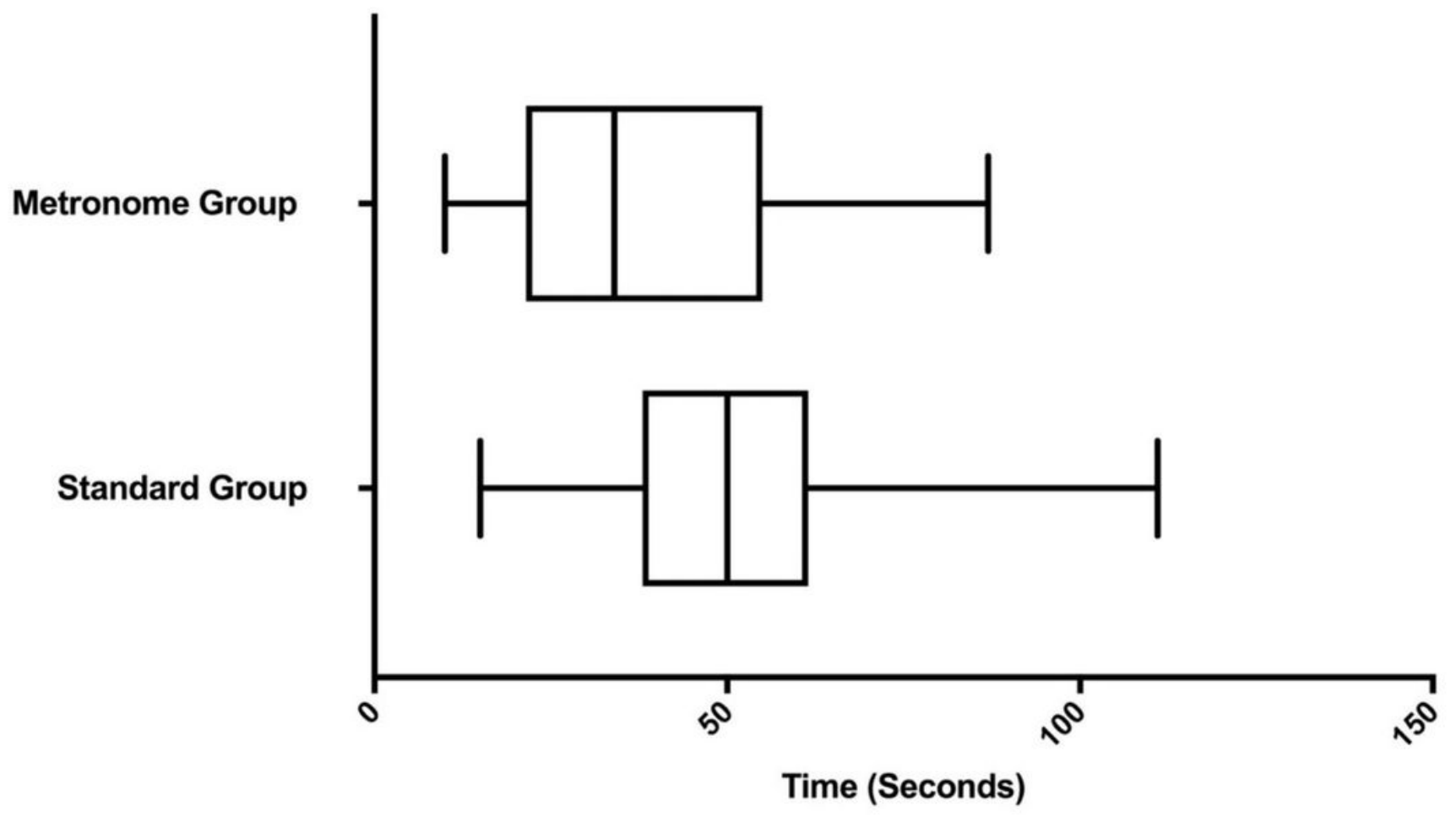

Figure 2

Box and whisker plots depicting median time to infusion rate goal. $\mathrm{P}=0.006$ 


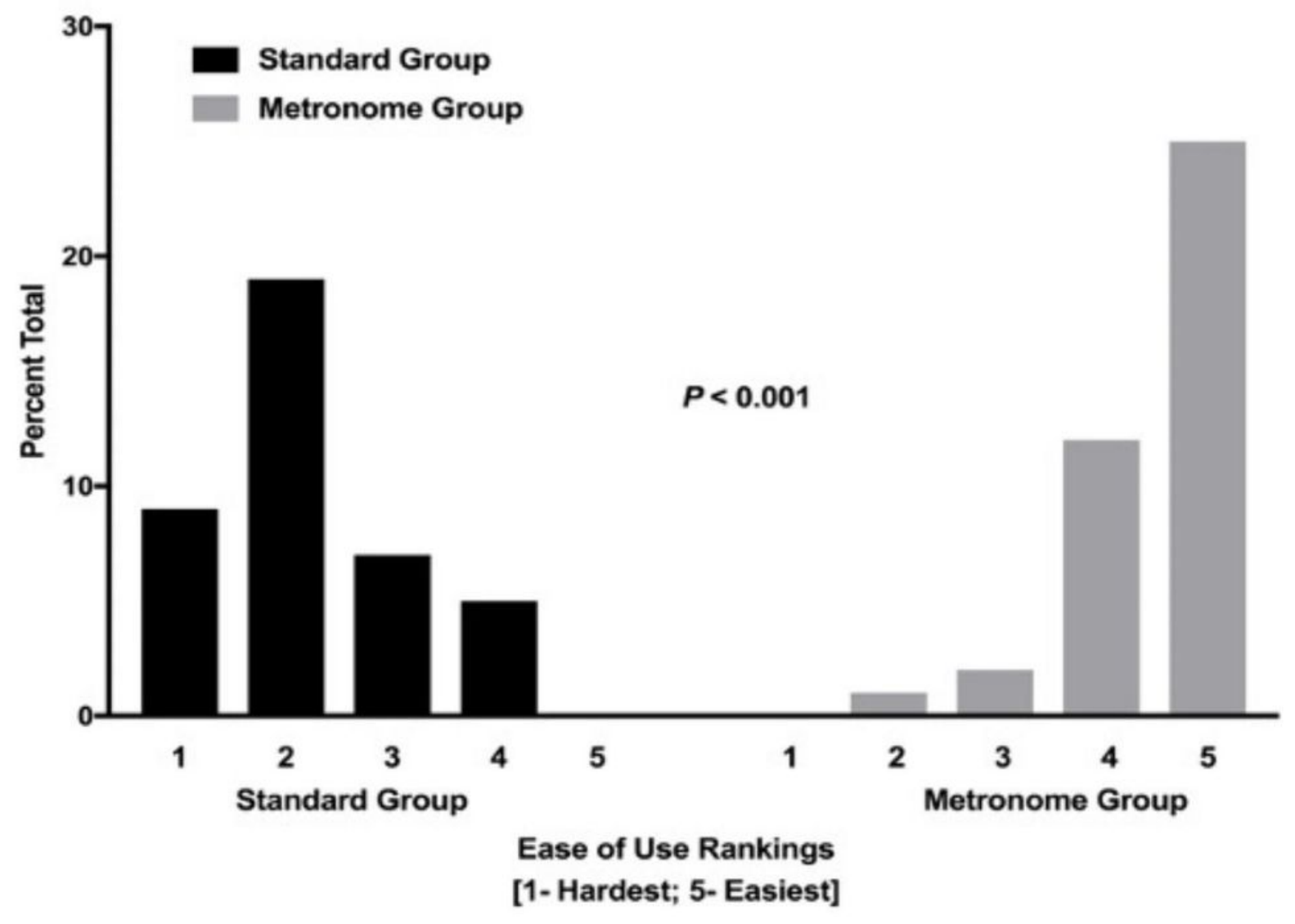

Figure 3

Self-reported ease of use 